\title{
Quand une rose n'en est pas une: petit traité de journalisme appliqué pour étudiants avancés
}

Le monde se complexifie. Cette constatation s'applique à de nombreux domaines de notre vie, y compris la médecine. Vous pensez bien sûr tout de suite à des notions comme médecine moléculaire, technologie génétique, nanotechnologie, robot da Vinci, «salvage therapy» ou tomographie à émission monophotonique ... Mais qui peut encore garder la vue d'ensemble, sans parler de celle de détail, sur les progrès technologiques fulgurants de notre temps?

Cette tendance n'épargne aucun domaine, même pas une activité relativement anodine comme la lecture d'une revue médicale. Au contraire, l'évolution dans ce secteur en marge de la médecine est pour le moins vertigineuse. Si une Gertrude Stein pouvait encore réduire les phénomènes de son époque à la formule «Une rose est une rose est une rose» et accéder à la célébrité grâce à cette tautologie plutôt banale, un manque aussi manifeste de créativité ne parvient aujourd'hui, dans le meilleur des cas, qu'à arracher un sourire fatigué aux faiseurs de journaux modernes.

Il n'y a que les éternels ploucs, comme nous à $\mathrm{EMH}$, pour s'en tenir, à la manière de Mme Stein, à la formule «Un article est un article est un article〉 ou 〈Une page de pub est une page de pub est une page de pub> et il convient de les signaler comme tels». La multifonctionnalité, une approche ludico-fantaisiste des formes journalistiques, est aujourd'hui de mise et, dans les salles de rédaction avant-gardistes, le futur a commencé depuis longtemps. L'Association zurichoise des transports publics a donné le ton avec une campagne publicitaire originale, en ornant les rames du RER d'un élégant paraphe qui annonçait «Je suis aussi un bateau». Plus tard sont arrivées des affiches, où l'on voyait un tram traversant le lac de Zurich ou un car postal avançant sur des rails, avec en-dessous l'inscription (traduction libre): «Un seul billet partout, tout en un. Vos Transports zurichois».

Tout en un: une devise que les éditeurs de revues médicales semblent avoir faite leur. En effet, pourquoi un article ne devrait-il être qu'un article, alors qu'il peut être en même temps une annonce payée? Quant à la loi universelle «Tout coule», elle peut aussi s'appliquer à la distinction entre les parties rédactionnelle et publicitaire des médias imprimés. Et l'on n'hésite pas à appliquer ladite loi, tout en renonçant élégamment contrairement à la campagne publicitaire précitée - à préciser «Je suis aussi une annonce financée par la firme XY». Résultat: dans les faits, le lecteur ne peut plus distinguer les articles payants de ceux qui ont été rédigés en toute indépendance.

Objection, votre Honneur, ceci est une distorsion malintentionnée de la réalité! Admettons. Il est clair que nous vivons à une époque où les médias attachent la plus grande importance à l'éthique et à la transparence. Tout lecteur ou lectrice qui se donne un minimum de peine pourra facilement faire la différence entre les deux sortes de texte.

Comment s'y prendre? Voici quelques astuces: dans les revues médicales, portez votre attention sur les éléments graphiques qui passent normalement inaperçus et sont placés de préférence en début ou en fin de texte, et comparez-les dans divers articles. Si vous tombez sur des formes différentes - par exemple des losanges et des ellipses - alors vous êtes sur la bonne piste! Feuilletez maintenant soigneusement la revue et arrêtez-vous sur les textes imprimés en tout petits caractères. Concentrez-vous sur la partie médiane du texte (oui, exactement là où votre regard ne se porterait jamais spontanément). Il y a de fortes chances que vous tombiez sur une phrase du genre: «Les articles payants sont désignés par un losange.» Eh bien, si ce n'est pas de la transparence, ça y ressemble drôlement!

La créativité ne connaissant aucune limite ou presque, il existe des variantes originales de cette façon d'assurer la transparence. Celles-ci représentent bien sûr un défi pour le lecteur, mais il convient de les accepter sportivement. La technique consistant à comparer les titres de rubriques est par exemple très prometteuse. $\mathrm{Si}$, dans une revue, vous êtes frappé par plusieurs titres semblables - du genre «Cardio-Flash» et «CardioFocus»-, c'est qu'une fois de plus vous avez du flair. Il vous suffit alors de suivre à la trace les petits caractères. Bonne chasse!

Bruno Kesseli 